\title{
Entre artes e saberes: sinos que encantam a cidade da fé
}

\author{
Between arts and knowledge: bells that enhance the city of faith \\ Entre las artes y el conocimiento: campanas que realzan la ciudad de la fe
}

Getuliana Sousa Colares

ORCID: https://orcid.org/0000-0001-5438-9494 Universidade Federal do Ceará, Brasil E-mail: getulianacolares@gmail.com Jonas Neri de Castro

ORCID: https://orcid.org/0000-0001-5587-3675 Universidade UNOPAR, Brasil

E-mail: jonascastro274@gmail.com

Maria Ivaneidiane Souza Colares ORCID: https://orcid.org/0000-0003-2364-8312 Universidade Estadual Vale do Acaraú, Brasil E-mail: duducolares_@hotmail.com

Ana Karolina Colares Nunes

ORCID: https://orcid.org/0000-0002-6654-2705 Universidade Federal do Ceará, Brasil E-mail: karolnunes@alu.ufc.br

Adriana Souza Colares Santos

ORCID: https://orcid.org/0000-0001-7922-6085 Universidade Estadual Vale do Acaraú, Brasil E-mail: adrianascolares@yahoo.com.br

Fábio Costa Santos

ORCID: https://orcid.org/0000-0001-7236-3917 Universidade Federal do Ceará, Brasil

E-mail: fabiocostasantos@yahoo.com.br

Antonia Jeruziana Souza Colares ORCID: https://orcid.org/0000-0002-7216-6339

Universidade Estadual Vale do Acaraú, Brasil E-mail: jerinha65123@gmail.com

Wendel Melo Andrade

ORCID: https://orcid.org/0000-0001-8921-7326 Universidade Federal do Ceará, Brasil

E-mail: professorwendelmelo@gmail.com

\begin{abstract}
Resumo
Este artigo apresenta um estudo sobre a trajetória de vida, saberes e artes, de Getúlio Colares Pereira, um cidadão que trabalhou com amor e dedicação como sineiro da Basílica de São Francisco das Chagas de Canindé-Ceará. Realizamos esta pesquisa de cunho bibliográfico tendo como fontes livros, artigos e documentos legais. O objetivo deste trabalho é estudar como foi a história de vida de Getúlio Colares Pereira, os seus ensinamentos e a arte desenvolvida nas badaladas do sino. Buscamos identificar os saberes que este sineiro proporcionou, mostrando suas conquistas e seus caminhos percorridos até alcançar o título de Tesouro Vivo e posteriormente o diploma de Mestre da Cultura pela Universidade Federal do Ceará (UFC). Concluímos através desta pesquisa que o conhecimento de mundo aliado a uma vida inteira de trabalho pode mudar a história de um ser humano. A cultura e os saberes deste Mestre da Cultura vêm cada vez mais sendo valorizada pelos estudiosos e pesquisadores. O Mestre da Cultura Popular Getúlio Colares Pereira, educou pelo exemplo, pelo amor ao que fez, pela fé, pela alegria com o que fez, pela persistência em fazer compartilhar o que sabe com os que se deixam cativar e, assim, os seus alunos aprendem tornando o tocar do sino uma cultura continua para as novas gerações.
\end{abstract}

Palavras-chave: Artes; Saberes; Sino.

\section{Abstract}

This article presents a study on the life trajectory, knowledge and arts, of Getúlio Colares Pereira, a citizen who worked with love and dedication as a bell ringer at the Basilica of São Francisco das Chagas in Canindé-Ceará. We carried out this bibliographic research using books, articles and legal documents as sources. The objective of this work is to study the life story of Getúlio Colares Pereira, his teachings and the art developed in the tolling of the bell. We seek to identify the knowledge that this bell ringer provided, showing his achievements and his paths to reach the title of Living Treasure and later the degree of Master of Culture by the Federal University of Ceará (UFC). We conclude through this research 
that knowledge of the world combined with a lifetime of work can change the history of a human being. The culture and knowledge of this Master of Culture are increasingly being valued by scholars and researchers. The Master of Popular Culture Getúlio Colares Pereira, educated by example, for the love of what he did, for faith, for the joy with what he did, for the persistence in making share what he knows with those who let himself be captivated and, thus, his students learn by making the ringing of the bell a continuous culture for new generations.

Keywords: Arts; Knowledges; Bell.

\section{Resumen}

Este artículo presenta un estudio sobre la trayectoria de vida, saberes y artes de Getúlio Colares Pereira, un ciudadano que trabajó con amor y dedicación como campanero en la Basílica de São Francisco das Chagas en Canindé-Ceará. Realizamos esta investigación bibliográfica utilizando como fuentes libros, artículos y documentos jurídicos. El objetivo de este trabajo es estudiar la historia de vida de Getúlio Colares Pereira, sus enseñanzas y el arte desarrollado en el tañido de la campana. Buscamos identificar los saberes que aportó este campanero, mostrando sus logros y sus caminos para alcanzar el título de Tesoro Vivo y luego el grado de Maestría en Cultura por la Universidad Federal de Ceará (UFC). Concluimos a través de esta investigación que el conocimiento del mundo combinado con una vida de trabajo puede cambiar la historia de un ser humano. La cultura y el conocimiento de esta Maestría en Cultura son cada vez más valorados por académicos e investigadores. El Maestro de Cultura Popular Getúlio Colares Pereira, educado con el ejemplo, por el amor de lo que hizo, por la fe, por la alegría con lo que hizo, por la persistencia en hacer compartir lo que sabe con quien se dejó cautivar y, así, sus alumnos aprenden haciendo del toque de campana una cultura continua para las nuevas generaciones.

Palabras clave: Artes; Conocimientos; Campana.

\section{Introdução}

A cultura nordestina vem sendo descoberta a cada dia, temos muitas belezas no sertão e aos poucos nós pesquisadores vamos tentando resgatar estas práticas existentes no Nordeste e em especial no Ceará.

Observamos que na cidade de Canindé-Ceará existem muitos sujeitos com talentos diversos, entre eles o sineiro Getúlio Colares Pereira que é um cidadão simples mais que teve um sonho de não deixar morrer um talento dado por Deus, talento este que lhe proporcionou tocar o sino do mais lindo santuário da Basílica de São Francisco das Chagas na cidade de Canindé-Ceará. Quem vem a esta cidade na época da festa do padroeiro sabe que as badaladas do sino chamam a atenção para a fé e a alegria da igreja, emocionando conterrâneos e romeiros que visitam a cidade.

Neste artigo, vamos conhecer um pouco desta linda história de fé, e desta longa trajetória de talento e encanto do sineiro Getúlio. Desse modo, esta pesquisa tem o objetivo de estudar como foi sua história de vida, os seus ensinamentos, a arte e saberes desenvolvidos nas badaladas do sino entoadas pelo Mestre da Cultura Getúlio Colares Pereira.

Notamos que este Mestre é um cidadão que trabalhou com amor e dedicação como sineiro da Basílica de São Francisco das Chagas em Canindé-Ceará durante 77 anos de sua vida. E isso tudo se iniciou com uma promessa da sua mãe desde seus 15 anos de idade.

Realizamos esta pesquisa de cunho bibliográfico tendo como fontes livros e artigos que retratam a vida e a trajetória deste sineiro e documentos legais relacionados ao registro de Mestre da Cultura.

Organizamos este artigo em seis seções, sendo a primeira esta introdução. Na segunda seção apresentamos o caminho metodológico desenvolvido nesta pesquisa. Na terceira seção discutimos a trajetória de vida de Getúlio Colares. Na quarta apresentamos as homenagens e prêmios recebidos por este sineiro. Na quinta tecemos algumas reflexões sobre os aspectos legais do registro dos Mestres da Cultura Tradicional Popular do estado do Ceará. E na sexta, tecemos nossas considerações finais.

\section{Caminho Metodológico}

Buscando o atendimento ao objetivo proposto, identificamos aqui uma pesquisa exploratória uma vez que seu planejamento é bastante flexível, de modo que possibilite a consideração dos mais variados aspectos relativos ao fato estudado. 
Com base nos escritos de Prodanov e Freitas (2013) a pesquisa exploratória tem por finalidade proporcionar mais informações sobre o assunto investigado, possibilitando sua melhor compreensão e delineamento, como também possibilitando estabelecermos novas perspectivas e enfoques ao assunto estudado.

Ademais, a pesquisa exploratória nos proporciona uma maior familiaridade com o assunto investigado, nos levando a compreender de uma forma mais ampla a trajetória de vida do Mestre da Cultura Getúlio Colares Pereira, enfatizando a sua história e os seus ensinamentos.

Em se tratando do procedimento metodológico, adotamos os pressupostos de uma pesquisa bibliográfica, por considerarmos um procedimento apropriado para o alcance do objetivo a ser alcançado. Para tanto, procedemos com um investigação em materiais já publicados, tais como, livros, artigos, documentos legais, entre outros.

Sobre este procedimento de pesquisa, Severino (2007) destaca que,

A pesquisa bibliográfica é aquela que se realiza a partir do registro disponível, decorrente de pesquisas anteriores, em documentos impressos, como livros, artigos, teses etc. Utiliza-se de dados ou de categorias teóricas já trabalhados por outros pesquisadores e devidamente registrados. Os textos tornam-se fontes dos temas a serem pesquisados. O pesquisador trabalha a partir das contribuições dos autores dos estudos analíticos constantes dos textos (Severino, 2007, p. 122).

A pesquisa bibliográfica é, portanto, um momento fundamental em todo trabalho científico pois estabelece os conceitos abordados na pesquisa, influenciando todas as discussões levantada durante as análises, possibilitando o estabelecimento de um embasamento teórico em que se apoia o trabalho (Prodanov \& Freitas, 2013).

Neste procedimento, a revisão bibliográfica deve trazer contribuições no desenvolvimento do conhecimento a ser investigado, trazendo mais significados ao fato estudado e colaborando com a evolução do trabalho (Sousa, 2021).

Nas seções seguintes apresentaremos os resultados e discussões oriundas das investigações e análise realizadas em decorrência deste procedimento metodológico.

\section{Trajetória das Artes e Saberes do Mestre da Cultura}

O Mestre da Cultura Getúlio Colares Pereira nasceu em 23 de março de 1929, na localidade de Alegre, distrito de Canindé-Ceará, e faleceu em 18 de março de 2021 em sua cidade natal, em decorrência de insuficiência respiratória ocasionada pelo contágio da doença causada por SARS-CoV-2, novo coronavírus (COVID-19).

No dia 21 de janeiro de 1944, com apenas 15 anos de idade, Getúlio começou a ajudar na igreja como acólito para pagar uma promessa que sua mãe havia feito. Ele deveria passar quatro anos ajudando na igreja.

A primeira vez que repicou foi em 29 de julho de 1944 com pouco mais de cinco meses que estava na igreja, por ocasião da Procissão do Coração de Jesus, pois a pessoa responsável por tocar o sino não pode comparecer e com isso Getúlio teve o primeiro contato com o objeto que, durante muito tempo iria ficar presente na sua vida, o sino. Mesmo sem nunca ter tocado, já teve sucesso na sua primeira vez, pois conseguiu fazer as badaladas no ritmo correto. A partir daí nunca mais abandonou essa atividade que tanto amou.

Getúlio, logo percebeu que, dependendo do motivo pelo qual seria necessário tocar o sino, as badaladas não poderiam ser sempre iguais. O som transmitido durante um enterro em que a tristeza é dominante não pode ser igual ao som transmitido numa festa onde a alegria comanda, assim começou a pensar em alguns modos diferentes sobre como poderia tocar o sino. Em estudos realizados pelo próprio Getúlio, foram identificadas 55 formas diferentes formas de tocar o sino, formas essas que vieram de vários países (Colares \& Adriana, 2017).

Getúlio Colares sempre ajudou na igreja como voluntário, apesar de ser o sineiro da Basílica de Canindé teve vários 
empregos, pois precisava trabalhar para poder se manter, já que era apenas voluntário na igreja. Um desses empregos foi o de coveiro no Cemitério São Miguel da sua cidade natal, onde passou 28 anos de sua vida. Em sua trajetória profissional, ele presenciou o enterro de 12.681 pessoas, entre crianças e adultos (Colares \& Adriana, 2017).

Mestre Getúlio foi casado por 47 anos com Maria de Jesus Sousa Colares. Foi pai de cinco filhas e avô de oito netos. Era aposentado, mas continuou a trabalhar até o fim da sua vida, sendo considerado um patrimônio vivo do Santuário de São Francisco das Chagas. Já que foi escolhido como um dos Tesouros Vivos do Ceará em 21 de Setembro de 2007, quando recebeu o diploma de Mestre da Cultura. Ele tornou-se conhecido em todo o país, chegando a conceder entrevistas há várias emissoras de rádio e televisão.

As culturas e as artes se encontram em caminhos ainda desconhecidos e incertos. A teoria e a práxis que até então buscavam-se recorrer ficam agora guardadas para tentar compreender as transformações culturais que a sociedade está passando, o que é sabido é que muita coisa mudou, principalmente quando considerado os novos caminhos artísticos e culturais e as novas formas de se consumi-los (Corá, 2021, p. 328).

A cultura brasileira é vasta e diversa. A nossa cultura pode ramificar-se em centenas de eixos, vindos dos vários polos culturais estabelecidos em todos os estados de todas as regiões, e em especial no Nordeste, no estado do Ceará e na cidade de Canindé, onde percebemos que o talento do mestre Getúlio Colares Pereira é inédito, pois suas badaladas são únicas e seus toques foram criados na prática, para cada ocasião e evento que era celebrado nesta cidade.

\section{Homenagens Recebidas pelos Sons do Sinos que Encantam a Cidade da Fé}

Em toda a sua trajetória de vida, o Mestre da Cultura Getúlio Colares Pereira recebeu diversas homenagens e prêmios em reconhecimento as suas contribuições como sineiro da Basílica de São Francisco das Chagas na cidade de Canindé-Ceará e ao seu valor para a história e a cultura nordestina. A seguir podemos observar algumas destas homenagens e prêmios.

- Recebeu seu primeiro prêmio uma medalha de prata, no dia 29 de Julho de 1946, quando tocou o Centenário de Canindé, ofertada pelo Dom Lustosa de Almeida, Arcebispo de Fortaleza;

- No dia 08 de dezembro de 1952, recebeu seu segundo prêmio, um anel de prata;

- Em 1954 recebeu uma medalha da Polícia Rodoviária Federal na pessoa do Inspetor Weber Sobral;

- Em 1958, recebeu medalha do Vigário da Paróquia de Pernambuco;

- Em 1959, pela Santa Sé de Fortaleza, Padroeira de Nossa Senhora de Assunção pela Catedral Metropolitana de Fortaleza;

- 1973 recebeu um crucifixo do Juiz da Comarca de Canindé, Dr. Oliveira;

- Em 1984 nos 50 anos de sineiro recebeu um relógio do Frei Batista Fernando Sobrinho;

- Em 29 de julho de 1999 recebeu da TV Verdes Mares um livro "Os Pés e o Sagrado";

- Recebeu também várias homenagens das Escolas CAIC, Frei Policarpo, IV Feira Estadual e de Ciências e Cultura da $7^{\mathrm{a}}$ CREDE em 2010; como também, IV Feira Regional e de Ciências e Cultura da $7^{\mathrm{a}}$ CREDE no mesmo ano;

- Em 2011, na comemoração dos 165 anos de Emancipação Política de Canindé recebeu do prefeito Claudio Pessoa uma medalha;

- Pelo reconhecimento do Dom artístico de sineiro recebeu homenagem do Colégio Paulo Sarasate em 30 de abril de 2002;

- Em 20 de fevereiro de 2003, Comenda Lojista CDL pelos 59 anos de trabalho prestado como sineiro;

- No dia 10 de outubro de 2004; recebeu da Paróquia de São Francisco um sino de bronze, pelos seus 60 anos de sineiro; 
- Em 16 de dezembro de 2005, Comenda da Escola Menino Jesus;

- Em 29 de julho de 2000, no $154^{\circ}$ aniversário do município de Canindé recebeu homenagem do prefeito Ximenes Filho;

- Diploma Mestre da Cultura, Tesouros Vivos do Ceará, certificado recebido em 21 de setembro de 2007 pelo governador do Estado do Ceará Cid Gomes;

- Troféu Francisco Xavier de Medeiros pelo radialista Chico Carloto;

- Homenagem da Escola Carlos Jereissati em 2011, "Projeto Folclore";

- Recebeu homenagem do Ministro Geral do Papa Bento XVI em 04 de junho de 2011;

- Universidade Federal do Ceará nomeado e outorgado Mestre da Cultura.

- Homenagem aos Mestres da Cultura do Projeto Paixão de Cristo.

- Homenagem no XIV Mostra Estadual Ciclo Natalino. Natal de Luz em dezembro de 2019.

\section{Aprovação para Compor Mestre da Cultura no Ceará}

A Secretaria da Cultura do Ceará baseada na Convenção para Salvaguarda do Patrimônio Imaterial da UNESCO, de 2003, definiu como “Tesouros Vivos da Cultura”, as pessoas, grupos e comunidades que são, reconhecidamente, detentoras de conhecimentos da tradição popular do Estado. Com isso, o Ceará deu um passo adiante em relação a outros estados brasileiros, sendo um dos pioneiros na preservação e proteção do seu patrimônio imaterial. Com a Lei no 13.351 de 27 de agosto de 2003 (Ceará, 2003), o Governo do Estado, através da Secretaria da Cultura SECULT garantiu o registro dos Mestres da Cultura Tradicional Popular, como forma de apoiar e preservar a memória cultural do povo cearense e o encargo de transmitir às gerações futuras o saber e a arte sobre os quais construímos a nossa história.

De acordo com Castro (2008),

As práticas representam expressões, conhecimentos e técnicas juntos com os instrumentos, objetos, artefatos e lugares culturais que lhes são associados que as comunidades, os grupos e, os indivíduos reconhecem como parte integrante de seu patrimônio cultural. Este patrimônio cultural imaterial que se transmite de geração em geração, é constantemente recriado pelas comunidades e grupos em função de seu ambiente, de sua interação com a natureza e de sua história, gerando um sentimento de identidade e continuidade e contribuindo assim para promover o respeito à diversidade cultural e à criatividade humana (Castro, 2008, p. 11).

A partir de 2005 a Secretaria da Cultura do Ceará começou a realizar encontros com os Mestres da Cultura, já foram oito encontros realizados, sendo seis, em Limoeiro do Norte, um em Juazeiro do Norte e o último no município do Crato. O primeiro que teve seis dias de duração e foi intitulado I Festival Mestres do Mundo, tinha como objetivo dar visibilidade aos Mestres da região do Vale do Jaguaribe.

Nesse encontro predominou os debates acadêmicos, durante o dia e a noite apresentações com os Mestres que vieram de vários estados e países como Japão, Portugal, Índia, México e Argentina. Foi um evento muito midiático com direito a encarte especial em jornal de grande circulação e mobilizou toda região principalmente as cidades envolvidas.

Segundo os organizadores, foi um sucesso de público e participação. A partir daí todos os outros encontros tiveram metodologia parecida, e uma programação com debates acadêmicos pela manhã, "rodas" de Mestres à tarde e apresentação culturais à noite. Mesmo os que foram realizados no Cariri. Passados oito edições do evento que se observa é que a participação da população local é muito pequena, além disso, não existe uma memória dos encontros, não existe nenhum documento que se possam aferir os indicadores, como a quantidade de Mestres que participaram, público presente, a participação das escolas do município dentre outros o que dificulta o planejamento dos próximos e continuasse com os mesmos erros.

Após a aprovação da Lei n ${ }^{\circ} 13.351$ (Ceará, 2003), que implanta uma política pública de promoção e preservação da 
Cultura Tradicional Popular no Ceará e de sua atualização através da Lei no 13.842 (Ceará, 2006), faz-se necessário fazer uma análise de sua aplicação.

Acreditamos que essa política é um grande avanço, o Ceará foi um dos pioneiros no Brasil, porém ela carrega durante esses anos vários problemas que até então não foram solucionados. A garantia da continuidade do saber e fazer do Tesouro Vivo, que segundo a Lei, é de responsabilidade do Mestre, a partir de um programa feito pela Secretaria da Cultura. Esta continuidade do saber e fazer do Tesouro Vivo envolve processos de ensino e aprendizagem e configura-se um desafio a ser enfrentado.

Assegurar a garantia da continuidade dos conhecimentos dos Metres da Cultura requer envolver a Secretaria de Educação do Estado e as Secretarias de Educação dos Municípios, para que esse programa possa ser implantado.

Há também a necessidade de se alterar a Lei no 13.842 (Ceará, 2006), retirando a limitação de apenas 60 Tesouros Vivos no Ceará, e isso se justifica pelo número de municípios que o estado tem, que são 184, e que em todos tem grupos e pessoas que se enquadram na legislação para concorrer ao título de Tesouro Vivo da Cultura, para que num futuro próximo possamos ter pelo menos um Tesouro Vivo por município, contemplando todas as regiões cearenses.

Com isso é importante inserir nesta Lei a regionalização do edital para que não ocorra a concentração de Mestres em uma determinada região em detrimento de outra. Também é necessário fazer uma grande sensibilização junto aos gestores culturais dos municípios com reuniões regionais mostrando a importância da política de promoção e preservação da Cultura Tradicional Popular antes de lançar os editais para que eles mobilizem seus Mestres e os incentivem a participar do certame.

Nesta perspectiva, faz necessário realizar um mapeamento mais qualificado do patrimônio material e imaterial dos municípios em parceria com as prefeituras, envolvendo alunos da rede pública. Esse mapeamento pode inclusive está articulado com as disciplinas extracurriculares para a continuidade dos conhecimentos dos Mestres.

Entre outras ações a serem desenvolvidas nesta direção, podemos destacar: Incentivar todos os municípios a implantar uma política de promoção e preservação da Cultura Popular Tradicional, criando seus conselhos de patrimônio, e reconhecendo seus Mestres com titulação municipal, mesmo que não tenha ajuda financeira; Promover uma maior participação dos Tesouros Vivos nos editais da Secretaria da Cultura; Apresentar projetos como demanda espontânea no Fundo Estadual da Cultura, transformar suas atividades em pontos de cultura; e Criar um sistema de documentação dos encontros e eventos já realizados.

Em 2006, a Lei no 13.351 (Ceará, 2003) foi revisada e ampliada, incluindo a manutenção dos grupos e coletividades. Publicada no Diário Oficial do Estado do Ceará, recebeu o nome de Lei dos Tesouros Vivos da Cultura com o número 13.842, de 27 de novembro de 2006 (Ceará, 2006).

As práticas representam expressões, conhecimentos e técnicas junto com os instrumentos, objetos, artefatos e lugares culturais que lhes são associados que as comunidades, os grupos e, os indivíduos reconhecem como parte integrante de seu patrimônio cultural.

Este patrimônio cultural imaterial que se transmite de geração em geração, é constantemente recriado pelas comunidades e grupos em função de seu ambiente, de sua interação com a natureza e de sua história, gerando um sentimento de identidade e continuidade e contribuindo assim para promover o respeito à diversidade cultural e à criatividade humana (Castro, 2008).

Este conceito nos dá a dimensão do caráter vivo deste patrimônio e da ação permanente do sujeito sobre ela, promovendo a sua interação como elemento fundamental para pensar políticas públicas de preservação e identificação de patrimônios vindouros. As políticas para preservação deste patrimônio precisam salvaguardar o já existente, sem negligenciar a ação de identificar e promover novos bens diante do conceito de cultura viva. A Constituição Federal de 1988, acerca da Cultura, estabelece que cabe ao Estado proteger as manifestações das culturas populares, indígenas e afro-brasileiras, e das de outros grupos participantes do processo civilizatório nacional (Brasil, 1988).

A Constituição Federal destaca ainda no Art. 216 que constitui o patrimônio cultural brasileiro de natureza material e imaterial, tomados individualmente ou em conjunto portadores de referência à identidade, à ação, à memória dos grupos 
formadores da sociedade brasileira (Brasil, 1988).

A Lei n 13.351 (Ceará, 2003) instituiu no Ceará, o Registro dos Mestres da Cultura Tradicional Popular do Estado. Para tanto o texto da Lei diz ser necessário que o interessado a concorrer ao título de Mestre da Cultura Tradicional Popular do Estado do Ceará/Tesouro Vivo, seja brasileiro, e residente no estado do Ceará há mais de 20 anos, que na data do seu pedido de inscrição possa comprovar sua participação em atividades culturais há mais de 20 anos, e ainda sejam capaz de transmitir seus conhecimentos e/ou suas técnicas a alunos ou aprendizes.

Portanto, o Mestre deve estar qualificado para propagar seus conhecimentos na produção manutenção e preservação da Cultura Tradicional Popular contribuindo para manutenção desta cultura em sua comunidade. A Lei n 13.351 de 2003 (Ceará, 2003) é composta por 18 artigos, nos quais analiso os artigos $3^{\circ}$ e $4^{\circ}$. O artigo $3^{\circ}$ da lei detalha os critérios para o processo de indicação de Registro dos Mestres da Cultura Tradicional Popular.

É necessário que o candidato tenha vida e obra relevantes para Cultura Tradicional Popular do Ceará; tenha reconhecimento público das tradições culturais desenvolvida; esteja em atividade com capacidade de transmitir seus conhecimentos artísticos e culturais; comprove sua experiência e vivência dos costumes e tradições culturais e por fim comprove a situação de carência econômica e social.

$\mathrm{O}$ artigo $4^{\circ}$ destaca os direitos que a pessoa natural passa a ter como o diploma de Título de Mestres da Cultura Tradicional Popular do Estado do Ceará; Auxílio financeiro vitalício de um salário mínimo mensal, deixando claro que esses direitos atribuídos ao Mestre diplomado são de natureza personalíssima, inalienáveis, e impenhoráveis, portando não podem ser cedidos e transmitidos a herdeiros ou legatários, assim como não geram vínculo de qualquer natureza com o Estado. Os referidos direitos se extinguem com o falecimento do mestre registrado.

Esta posição corrobora com o a premissa de que os Mestres da Cultura têm um papel fundamental nos ensinamentos e na continuidade dos saberes da cultura popular, de que são conhecedores e que se traduzem numa contribuição para a educação patrimonial, que neste caso em estudo, mais relacionado com o patrimônio imaterial.

O Mestre Getúlio Colares, portanto, como um Mestre da Cultura Tradicional Popular de seu estado, contribuiu, com a sua prática, com o seu ofício de sineiro e de Mestre conhecido mundialmente, com a manutenção, a divulgação, a revitalização destas duas atividades culturais e, por conseguinte, com a educação patrimonial imaterial, no seu município e no raio de abrangência de sua atividade artística e cultural. Sua contribuição, por vezes é diretamente junto aos estudantes, quando estes o procuram para dar depoimentos sobre a cultura, sobre suas atividades e experiência, ou ainda quando participava de palestras, debates, entrevistas, discussões em salas de aula, e de pesquisas em Trabalhos de Conclusão de Curso (TCC), dissertações, teses, artigos ou em outras atividades acadêmicas.

No entanto a sua contribuição junto ao processo de educação patrimonial, de caráter imaterial, se dá de maneira mais atuante, fora dos muros da escola, desde quando participou das primeiras apresentações na década de 1940 como relata em seus depoimentos, ou seja ao longo de sua vida. Na Secretaria da Cultura não existe nenhum documento que possa evidenciar a intenção de se elaborar alguma parceria com a Secretaria de Educação no sentido de implementar um programa de ensino nas escolas públicas do Estado. Para o atual Coordenador de Patrimônio Histórico e Cultural da SECULT, Otávio Menezes, Coordenador de Núcleo de Patrimônio Imaterial da SECULT 2003 a 2007:

Há entraves burocráticos por parte de setores educacionais para entender a posição do Mestre na escola. Uma espécie de força ideológica contrária baseada na opinião de que, por exemplo, como pode um Mestre da Cultura, sem escolaridade, muitas vezes não saber sequer assinar o nome, ensinar algo? Assim, o saber adquirido pela informalidade não interessa. Como se a função do Mestre da Cultura na Escola fosse ensinar a reproduzir manifestações da cultura subalterna (indígena, negra) a qual, embora haja um enorme esforço para compreendê-la, dentro da nossa historicidade, ainda, lamentavelmente, é motivo de preconceitos. Como se os Tesouros/Mestres fossem disseminar produtos de uma economia atrasada, superada. Por outro lado, a Secretaria da Cultura não insistiu no sentido de criar um programa de 
aproveitamento do Tesouro/Mestre na escola, com uma metodologia específica e parcialmente desvinculada da grade curricular do ensino formal. (Menezes, 2007 apud Martins, 2011, p. 6,).

Buscando valorizar a cultura nacional o Ministério da Cultura, instituiu a Emenda Constitucional (EC) n ${ }^{\circ} 48$, de 10 de agosto de 2005 (Calabre \& Zimbrão, 2020). Essa EC implementou o Plano Nacional de Cultura (PNC), com previsão de duração plurianual, objetivando o desenvolvimento cultural do País e a integração das ações do poder público.

Queiroz (2019) constrói uma linha histórica sobre a elaboração e institucionalização do Sistema Nacional de Política Cultural desde o final dos anos 1990, até culminar em 2010, com a aprovação da lei do Plano Nacional de Cultura (2010-2020) que previa para o Brasil em 2020:

Imaginar o cenário da cultura em 2020 é pensar que até lá o povo brasileiro terá mais acesso à cultura e que o país responderá criativamente aos desafios de cultura do nosso tempo (...) até 2020 as políticas culturais terão passado por diversas transformações (...) serão desenvolvidas políticas para fortalecer a relação da cultura e áreas como educação, a comunicação social, o meio ambiente, o turismo, a ciência e tecnologia e o esporte. (...) A concepção da cultura passa a compreender uma perspectiva ampliada na qual se articula três dimensões: a simbólica, a cidadã e a econômica (Brasil, 2010).

Sabemos que a cultura é de suma importante, pois é a afirmação da identidade de uma nação, visto que composta por todos os valores, símbolos e costumes de um povo. Para que, dessa forma, seja construído a identidade cultural dos povos mostrando a diversidade cultural.

Artistas das diversas áreas também passaram a buscar, a reivindicar, formas de participar e de interferir nos processos de decisão no campo das políticas públicas culturais. Ressurgiram movimentos de valorização das manifestações culturais locais, que incentivaram tanto a redescoberta de artistas da comunidade como de novos atores e formas de produção artístico-culturais (Calebre, 2018, p.43).

Portanto, os incentivos culturais, estimulam o desenvolvimento e proporcionam acessibilidade à cultura, oportunizando trocas de saberes e a descoberta de diversidades culturais. Divulgando as culturas e saberes, que ainda se encontram escondidos em nosso pais (Brasileiro et al. 2021).

Nesta breve síntese, podemos perceber um pouco das ações de fomento à cultura brasileira e a trajetória do Mestre Getúlio Colares Pereira, onde podemos conhecer as conquistas e desafios vivenciados por este sineiro, e principalmente do legado deixado por ele para a cultura cearense e nacional.

\section{Considerações Finais}

O Mestre Getúlio Colares Pereira, foi sineiro e um dos símbolos marcantes da festa de São Francisco da Chagas em Canindé-Ceará. Entoando o som das batidas do sino da Basílica de deste município ele fez história. Os devotos e romeiros conhecem de longe o bonito som que foi a sua marca registrada.

De longe, ao escutar o soar do seu sino, o romeiro para. É hora de rezar! E quem foi o responsável por esse momento de oração que desde de 1944 encantou e embalou o Santuário de Canindé foi o Mestre da Cultura Getúlio Colares, que durante o dia subia o sino 5 vezes no alto da torre da Basílica para tocar os sinos centenários. Os sons emocionavam os romeiros e a todos da região que vinham até a cidade.

Portanto percebemos que os sons emitidos pelas badaladas dos sinos da Basílica de Canindé-Ceará abrilhantavam a cidade nos períodos festivos. Com isso, o Mestre da Cultura Getúlio Colares Pereira, emocionou muitos devotos de São Francisco das Chagas em Canindé-Ceará, com seus lindos repiques que encantaram não só esta cidade do interior do Ceará, mais todo o estado e porque não dizer toda a nação. 
A relevância e a função social do trabalho com o patrimônio documental levam-nos a concluir que políticas de ação cultural que se aproximam da educação patrimonial como metodologia são fundamentais nos programas de difusão cultural no sentido de popularizar os arquivos, de compreendê-los para além de suas funções burocráticoadministrativas, tomando-os como instituições de informação, de preservação de direitos subjetivos e de memória. (Barcellos \& Sousa, 2020, p. 252).

Portanto, registramos saberes que ficarão marcados pelo tempo, nas lembranças, nas leituras, reavivando momentos que ficarão registrados na eternidade. Podemos eterniza às artes e às culturas, e conseguir representá-lo nas escritas, tornando-se o registro, por meio de diversas linguagens artísticas e manifestações culturais.

Concluímos que é de suma importância, preservar o Patrimônio Cultural, trouxemos neste artigo, artes e saberes que encantam a cidade de Canindé. Consideramos que o mesmo foi alcançado através da pesquisa bibliográfica, passeando por diversos momentos do chão vivido pelo mestre in memória Getúlio Colares Pereira que eternizou suas badaladas sonoras feita com todo amor e dedicação a sua profissão de sineiro da Basílica de São Francisco das Chagas de Canindé, no Ceará.

\section{Referências}

Barcellos, B. G. B. \& Sousa, E. G. (2021). Difusão cultural e educação patrimonial nos arquivos: semana nacional de arquivos. Anais XI Seminário internacional de politicas cuturais. Niteroi, RJ, Brasil, sescsp.org.br/uploads/BibliotecaTable/9c7154528b820891e2a3c20a3a49bca9/342/16226713421849347275.pdf

Brasil. (1938). Decreto Lei $n^{o}$. 526, de $1^{o}$ de Julho de 1938. Institui o Conselho Nacional de Cultura. http://www2.camara.leg.br/legin/fed/declei/19301939/decreto-lei-526-1-julho-1938-358396-publicacaooriginal-1-pe.html

Brasil. (1988). Constituição da República Federativa do Brasil. Senado Fedaral: Centro Gráfico.

Brasil. (2005). Emenda Constitucional $n^{o}$. 48, de 10 de agosto de 2005 . Instaura o Plano Nacional de Cultura. http://www.planalto.gov.br/ccivil_03/constituicao/emendas/emc/emc48.htm

Brasil. (2010). Decreto Lei. $n^{o}$. 12.343 /2010. Plano Nacional de Cultura - PNC e Sistema Nacional de Informações e Indicadores Culturais - SNIIC. www.planalto.gov.br/ccivil_03/_ato2007-2010/2010/lei/112343.htm

Brasil. (2013). As Metas do Plano Nacional de Cultura. https://www.ipea.gov.br/participacao/images/pdfs/ conferencias/IIICNCultura/metas-do-plano-nacionalde-cultura.pdf.

Brasil. (2020). Decreto Lei $n^{o} .14017$ de 25 de agosto de 2020. Ações emergenciais destinadas ao setor cultural a serem adotadas em decorrência dos efeitos econômicos e sociais da pandemia da covid-19. https://www.camara.leg.br/ proposicoesWeb/fichadetramitacao?idProposicao=2242136

Brasileiro, S. H. et al. (2021). Noções introdutórias sobre patrimônios históricos, artísticos e culturais. Research, Society and Development, 10(6), e31910615781, 2021, https://rsdjournal.org/index.php/rsd/article/view/15781/14129

Sousa, O. A. (2021). A pesquisa bibliográfica: princípios e fundamentos. Cadernos da Fucamp, 20(43), 64-83. https://www.fucamp.edu.br/editora/index.php/cadernos/article/view/2336

Calebre, L. \& Zimbrão, A. C. S. PNC 2010-2020 - um olhar sobre os percursos. Revista Observatório 28. São Paulo: Itaú Cultural. https://issuu.com/itaucultural/docs/observatorio29

Calebre, L. (2018). Cultura, territorialidade e direitos: a gestão municipal de cultura. In Carneiro, J. \& Baron, L. (Orgs.), Gestão Cultural. Niterói Livros.

Castro, M. L. V. (2008). Patrimônio imaterial no Brasil. UNESCO, Educarte.

Ceará. (2003). Lei $n^{o}$. 13.351, de 22 de agosto de 2003. Diário Oficial do Estado do Ceará. Fortaleza. https://belt.al.ce.gov.br/index.php/legislacao-doceara/organizacao-tematica/cultura-e-esportes/item/3346-lei-13-351-de-22-08-03-d-o-de-25-08-03

Ceará. (2006). Lei $n^{o}$. 13.842, de 27 de novembro de 2006. Diário Oficial do Estado do Ceará. https://www.al.ce.gov.br/legislativo/legislacao5/leis2006/13842.htm

Corá. (2021). Reflexões acerca das culturas e das artes em tempo de pandemia. Revista NAU Social, 11(21), $321-329$.

Colares, S. G., \& Adriana, S. C. S. (2017). Memória de um tesouro vivo. Encontro Internacional de Jovens Investigadores. Brasília. 2017. https://www.editorarealize.com.br/editora/anais/join/2017/TRABALHO_EV081_MD1_SA144_ID2219_15092017184337.pdf

IPHAN. (2007). Instituto do patrimônio histórico e artístico nacional - IPHAN. http://portal.iphan.gov.br/ pagina/detalhes/29

Martins, K. D. (2011). Culturas popular e erudita, breve revisão. Revista de Ciências Humanas, 11(2), 235-244. https: //www.cch.ufv.br/revista/pdfs/artigo2vol11-2.pdf.

Prodanov, C. C. \& Freitas, E. C. (2013). Metodologia do trabalho científico: métodos e técnicas da pesquisa e do trabalho acadêmico. (2a ed.), Feevale.

Queiroz, I. A. (2019). A arquitetônica da esfera política-cultural brasileira nos enunciados do Sistema Nacional da Cultura. (Tese de doutorado). Faculdade de Filosofia e Ciências Humanas da Universidade de São Paulo, São Paulo. https://www.teses.usp.br/teses/disponiveis/8/8142/tde-15082019-093817/pt-br.php 